thin depletion area at the surface, as Bardeen thought might be the case.

Shive's demonstration caused Shockley to propose an important modification of the original transistor. Instead of injecting and collecting minority carriers with the use of point contacts, one could employ relatively large area $\mathrm{p}-\mathrm{n}$ junctions and gain the advantage of much larger currents. The concept of the bipolar junction transistor, which was to dominate the field for the next two decades or so, was born. A semiconductor triode was not only feasible but was about to generate one of the greatest technological revolutions in history.

The text is rich in anecdote, presenting the reader with colourful views of many of the secondary as well as the primary figures involved. Backgrounds, hopes and destinies are examined. Matters such as Shockley's gradual change in personality as he became a celebrity, and the changes in the working atmosphere which made Bardeen decide to return to an academic career, are dealt with in a forthright manner.

Although the book is written in a semipopular style which should appeal to the type of general reader interested in the history of technology, the historical research on which it is based is impeccably sound, unlike many books of this kind. The authors, with their professional reputations involved, have left no stone unturned in making their work an enduring classic.

Frederick Seitz is at the Rockefeller University, 1230 York Avenue, New York,

New York 10021-6399, USA.

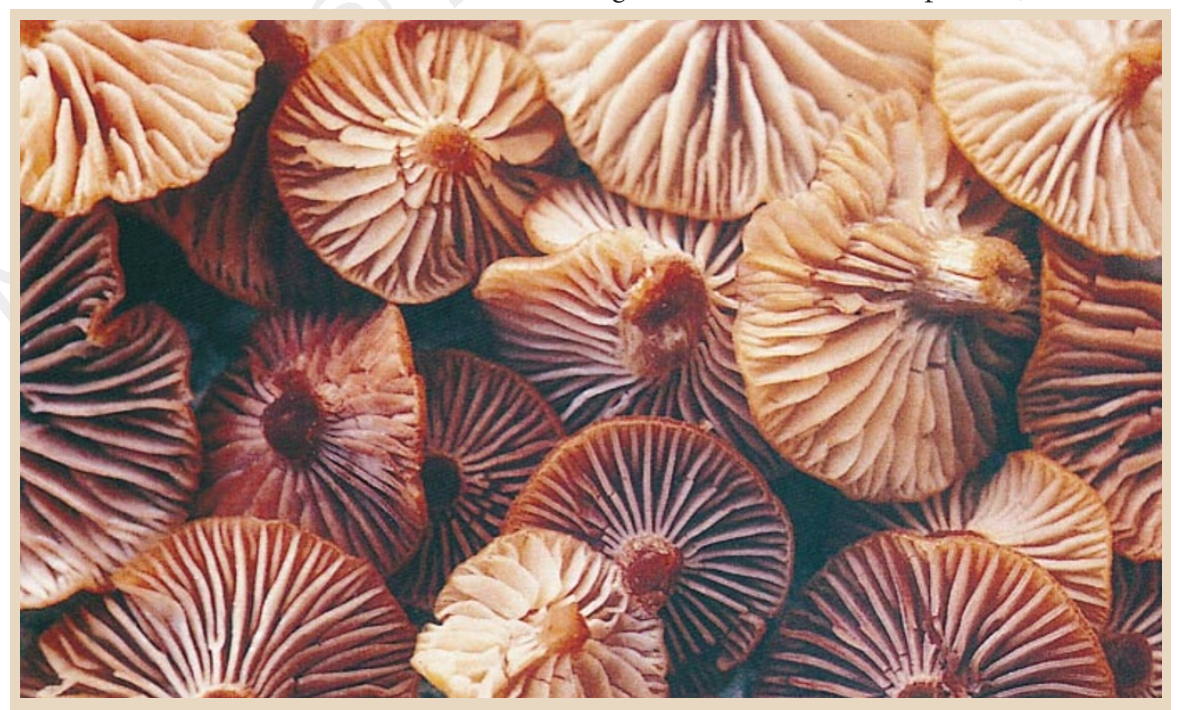

\section{The magic of mushrooms}

Life may be too short to stuff a mushroom, as the writer Shirley Conran said, but make time to read all about them in Elio Schaechter's funny and fascinating book, In The Company of Mushrooms: A Biologist'sTale (Harvard University Press, $\$ 24.95, £ 16.50$ ). Fungi have been found in fossilized wood 300 million years old, yet they are more closely related to animals

\section{Ceaseless}

\section{fluctuations}

\section{Conceptual Developments of} Twentieth Century Field Theories

by Tian Yu Cao

Cambridge University Press: 1997. Pp. 433

$\mathfrak{E} 45, \$ 59.95$

\section{lan Aitchison}

A century can be a convenient unit of history. During the past hundred years or so, quantum field theory has replaced classical mechanics as the framework of fundamental physics. The climax of this development has been the spectacularly successful Standard Model, a relativistic quantum gauge field theory describing matter and forces at the smallest sizes currently accessible.

But the intricate theoretical structure has imperfections, and the general quantum field framework has failed to accommodate gravity on the same footing as the other known forces. There are tantalizing hints of a new physics beyond quantum field theory. It seems a good moment to take stock.

Tian Yu Cao's timely book provides a broad overview of twentieth-century field theories. The story is presented in three parts, each devoted to a general line of development which he subsumes under the heading of a "research programme".

The first part covers the geometrical programme (Einstein's metric field theory of gravitation, and its developments); the second than to plants. And there's no shortage of them: Schaechter estimates that there are about two tons of fungi for every human being on Earth. Vital in the kitchen, fungi play an even more important role in recycling. "Life on earth as we know it would be impossible without them," he says. So we'll have to forgive them for Dutch elm disease and the Irish potato famine. concerns the quantum field programme (the emergence, formulation and application of quantum field theory, including current algebra, anomalies, renormalization and the renormalization group); and the third describes the gauge field programme (the Standard Model, and some extensions). The discussion is on occasion necessarily technical, but this is not an advanced physics textbook: rather, it is an up-to-date, well informed and detailed historical account of the conceptual origins and development of twentiethcentury field theories, structured in terms of the above programmes.

This is not all, however. Surrounding this substantial historical meat are introductory and closing chapters that are philosophical in character. For me, they are the most stimulating parts of the book.

Cao's aim is to show that there is a pattern in the conceptual evolution that he describes. In particular, he is concerned to combat the claim, originating in the work of Kuhn and others, that the history of science reveals no coherent direction of ontological development, and hence that no theoretical ontology can be taken as the real ontology of the world. Or, in other words, no matter how empirically successful our succeeding theories may be, they do not provide access to metaphysical reality. The contrasting position that Cao wishes to defend is characterized by him as "structural realism" which, roughly speaking, holds that the structural relations between entities (often expressed by mathematical structure) in a successful theory should be taken as real, rather than the entities themselves.

For this enterprise to succeed, Cao must show that structural properties of theoretical ontologies do persist across conceptual revolutions. This requirement, together with a desire to defend the rationality of scientific growth, leads him to propose that scientific revolutions can occur by what he calls "ontological synthesis", in which an outmoded ontology survives as an epiphenomenon, derivable from a later and more fundamental ontology. Like most physicists, I am all in favour of realism and rationality, but, while I applaud Cao's attempt, I think his analysis is open to serious challenge.

Classical field theory arose from the need to explain how physical actions, such as gravity and electromagnetism, can apparently be transmitted between bodies some distance from each other; this is in contrast to mechanical actions, which occur by direct contact. Newton hated the idea of action at a distance; and even Maxwell, whose equations for the dynamics of the electromagnetic field are now part of the Standard Model, thought his equations described the dynamics of a mediating medium, the ether, which he took to be some sort of mechanical substance. The ontological shift, by which the field became established as a non-mechanical entity in its 


\section{In retrospect chosen by John L. Casti}

\section{Gödel, Escher, Bach: An Eternal \\ Golden Braid}

by Douglas Hofstadter

(1979)

A classic dinner-party game I like to play is to ask my guests what science book published, say, during the past 25 years they would take if they were to be stranded indefinitely on a desert island. For me the choice is easy: I would grab a copy of Douglas Hofstadter's Gödel, Escher, Bach (GEB) before abandoning ship.

Alternating witty, enlightening dialogues with chapters on such meaty topics as selfreference, recursion, meaning in mathematics, formal systems of logic, brains, the weird and wonderful art of M. C. Escher, minds, thoughts, typesetting, Gödelian paradoxes, selfreplication, artificial intelligence and hierarchies, GEB is more like a summary statement of most of modern cognitive science than a thematic book, more of a romp over the modern intellectual landscape than a scholarly monograph. Yet it manages to be all these things and more.

At the time $G E B$ was sitting on bestseller lists around the world, a lot of people joked that it was a book everyone bought and no one read. Well, not me. I have a friend who says that each Christmas he rereads every Sherlock Holmes story because he learns something new with each rereading. I don't want to claim that I've now reread GEB 17 times, but I have read it cover-to-cover several times - and always with great profit. One reading taught me things about Bach fugues that I had never properly

own right, is very drastic, and Cao does not explain how a mechanical ontology can emerge from field physics as an epiphenomenon. Perhaps we just have to accept an ontological enlargement.

The situation is much worse, I believe, in the case of quantum field theory. Superficially, quantum field theory sounds like a synthesis of quantum mechanics and classical field theory. But this immediately leads to a problem. The quanta of force fields are bosons, yet the theory must also include fermions, for which there is no classical field. As Cao explains, this led historically to the procedure called "second quantization", which seems to be associated with a particle rather than a field ontology. But either ontology (particle or field) is problematic, because bosonic and fermionic quantum fields are quantum operators whose matrix elements are quantities from which only probabilities can be calculated, as is typical in quantum mechanics.

Cao claims that physicists have still not clarified the "real material" versus "probabilistic" character of the quantum field. He himself regards it as a new kind of substantial field, and with this view it is easier to find the kind of ontological continuity he wants. For appreciated, another time I gained a deeper insight into the figure-ground distinction, while a third reading heightened my sensitivities to the connection between Gödel's “incompleteness theorem”, self-reference and Escher's famous engraving Ascending and Descending. All these intellectual gleanings are secondary matters to the book's main theme, which is an extended meditation on the possibility of duplicating human thought processes in a machine.

I don't think it would be too great a claim to date the birth of the 'connectionism' movement in artificial-intelligence research to the publication of GEB in early 1979. This is the view that the physical structure of the human brain matters when it comes to trying to duplicate in a computing machine what the brain does.

Before then, workers had spent the better part of 30 years trying to skim off human intelligence from the actual physiological structure of the brain, essentially ignoring things like behaviour and connective structures at the level of the brain's neurons. Hofstadter redirected attention to these lower-level structures, arguing that if one wants to capture human cognition in a mechanical intelligence, the machine must mirror these low-level features of the brain. $G E B$ 's presence on the bestseller lists for months, along with its winning the 1980 Pulitzer prize for general nonfiction, brought this connectionist view of machine intelligence back to the forefront of research in artificial intelligence. Thus were reborn the theories of neural networks, genetic

example, he "can claim without hesitation that the gauge field programme is a direct descendant of the geometrical programme".

In fact, quantum field theory is really the result of trying to reconcile quantum mechanics with special relativity. Its ontology is no different from that of quantum mechanicsitself — and here we hit a barrier. Ontology, Cao says, is "concerned with an autonomous existence without reference to anything external". But, according to what is still the standard (Bohrian) view, although the existence of some independent microscopic reality behind observed phenomena is accepted, questions about the nature or state of that reality have meaning only in the context of explicitly stated external experimental setups. In some way, attributes and quantities even entities themselves - are ceaselessly fluctuating at the quantum level, and become real only as a result of 'measurement'. The simple connection between observed properties and ontological properties of an independently existing object has been severed. Of course, other interpretations have been proposed such as the 'many worlds' one; but this example has rather too much ontological baggage for many people's taste. algorithms, evolutionary programming and all the other 'hot' theories in the field.

It is interesting to ponder what makes $G E B$ a book many purchasers have never read, while it has changed other folk's view of the world like mine. On the downside, the book is a real doorstop, 778 pages long and more than two pounds in weight. So it's a bit of a package for comfortable reading on the subway or the beach. Some people have complained that it is rather disjointed and rambling, moving from topic to topic and back again with no apparent beginning or end. While I have to admit that there is some truth to these allegations, one might register the same complaints about the Encyclopedia Britannica. GEB cannot be measured by the standards usually employed to evaluate books. It's a one-of-a-kind volume that has to be looked at in its own right, which to their eternal credit the Pulitzer committee members immediately recognized.

On the asset side of the ledger, GEB is the best example I know of a 'transdisciplinary' book. Laying out explicit linkages between the humanities, art, science and mathematics, it proves that the landscape of the intellect does not come conveniently packaged into compartments such as English, physics, computer science and psychology. In some ways, GEB is an entire humanistic education between the covers of a single book. So, for my next visit to a desert island, give me sun, sand, water and $G E B$, and I'll live happily ever after.

John L.Casti is at the Santa Fe Institute, Santa Fe, New Mexico 87501, USA.

Perhaps Cao should have avoided the 'substantial' aspect of ontology, and stuck to the 'structural' aspects he himself advocates: witness, for example, the mathematical survival of Maxwell's equations. But, even at this level, the significance of the mathematical similarity between the space-time geometry of general relativity and 'internal' symmetries of gauge field theory is unclear.

Despite their having been invented in the same century, there is still a great divide between general relativity and quantum field theory. Perhaps the difficulty of reconciling them is an indication that our current ontological categories are inadequate, and that Cao's bold attempt at ontological synthesis is simply premature.

Ian Aitchison is in the Department of Physics, University of Oxford, 1 Keble Road, Oxford OX1 3NP, UK.

\section{correction}

The title of the book by Harrison G. Pope Jr reviewed by Stuart Sutherland in last week's issue (Nature 388, 239; 1997) was given incorrectly. It is Psychology Astray: Fallacies in Studies of 'Repressed Memory' and Childhood Trauma. 